\title{
Targeted vessel reconstruction in non- contrast-enhanced steady-state free precession angiography
}

\author{
Efe Ilicak ${ }^{a}$, Suheyla Cetin ${ }^{b}$, Elif Bulut ${ }^{c}$, Kader Karli Oguz ${ }^{c}$, \\ Emine Ulku Saritas ${ }^{a}$, Gozde Unal ${ }^{d}$ and Tolga Çukur ${ }^{a *}$
}

\begin{abstract}
Image quality in non-contrast-enhanced (NCE) angiograms is often limited by scan time constraints. An effective solution is to undersample angiographic acquisitions and to recover vessel images with penalized reconstructions. However, conventional methods leverage penalty terms with uniform spatial weighting, which typically yield insufficient suppression of aliasing interference and suboptimal blood/background contrast. Here we propose a two-stage strategy where a tractographic segmentation is employed to auto-extract vasculature maps from undersampled data. These maps are then used to incur spatially adaptive sparsity penalties on vascular and background regions. In vivo steadystate free precession angiograms were acquired in the hand, lower leg and foot. Compared with regular nonadaptive compressed sensing (CS) reconstructions $\left(\mathrm{CS}_{\mathrm{low}_{\mathrm{ow}}}\right)$, the proposed strategy improves blood/background contrast by $71.3 \pm 28.9 \%$ in the hand (mean \pm s.d. across acceleration factors $1-8$ ), $30.6 \pm 11.3 \%$ in the lower leg and $28.1 \pm 7.0 \%$ in the foot (signed-rank test, $\boldsymbol{P}<\mathbf{0 . 0 5}$ at each acceleration). The proposed targeted reconstruction can relax trade-offs between image contrast, resolution and scan efficiency without compromising vessel depiction. Copyright $\odot 2016$ John Wiley \& Sons, Ltd.
\end{abstract}

Keywords: sparsity; regularization; angiography; segmentation; vasculature; compressed sensing

\section{INTRODUCTION}

Non-contrast-enhanced MR angiography (NCE MRA) offers great potential in monitoring of atherosclerotic diseases, because it prevents complications due to contrast agents leveraged in routine contrast-enhanced (CE) examinations (1). Various successful approaches have been proposed to acquire NCE angiograms, including time-of-flight angiography, phase-contrast angiography, fresh-blood imaging and flow-independent angiography (FIA) (2). While these methods offer sensitive assessments of vessel morphology, image quality may be compromised due to limitations on scan time.

In the case of FIA, blood is delineated based on intrinsic $T_{1,2}$ and chemical shift differences among tissues. FIA employs magnetization-prepared, segmented steady-state free precession (SSFP) sequences to generate blood-background contrast $(3,4)$. This preparation overhead reduces scan efficiency and limits the achievable contrast and resolution (5), which is a concern for many other NCE methods as well (2). Note that limited contrast levels due to unwanted interference from background tissues can degrade the quality of vessel depiction severely. Therefore, acceleration strategies that improve scan efficiency while suppressing background signals can greatly increase the clinical potential of NCE MRA.

Due to the inherent structural sparsity of angiograms, acceleration can be achieved through undersampling followed by sparse reconstructions (6-10). To suppress aliasing artefacts and noise, penalties are applied typically based on $\ell_{1}$-norm or spatial finite differences of reconstructed images $(6,8,11,12)$. Relative weighting of penalties with respect to a data consistency term is a critical determinant of image quality in these reconstructions (6). Small weights can lead to insufficient artefact suppression and elevated background signals, whereas large weights can cause loss of relatively small or low-contrast vessel signals (8). This results in a fundamental compromise between blood-background contrast and vessel preservation.

\footnotetext{
* Correspondence to: T. CCukur, Department of Electrical and Electronics Engineering and the National Magnetic Resonance Research Center, Bilkent University, Bilkent, Ankara, Turkey.

E-mail: cukur@ee.bilkent.edu.tr

a E. llicak, E. U. Saritas, T. Çukur

Department of Electrical and Electronics Engineering and the National Magnetic Resonance Research Center, Bilkent University, Ankara, Turkey

b S. Cetin

Faculty of Engineering and Natural Sciences, Sabanci University, Istanbul, Turkey

c E. Bulut, K. K. Oguz

Hacettepe University Faculty of Medicine, Ankara, Turkey

d G. Unal

Department of Computer Engineering, Istanbul Technical University, Istanbul, Turkey
}

Abbreviations used: $2 D$, two-dimensional; $3 D$, three-dimensional; $C E$, contrast-enhanced; $C S$, compressed sensing; FIA, flow-independent angiography; FWHM, full-width at half-maximum; MIP, maximum-intensity projection; $M R A$, magnetic resonance angiography; NCE, non-contrast-enhanced; PSF, point-spread function; ROI, region of interest; SNR, signal-to-noise ratio; SSFP, steady-state free precession; TCS, targeted compressed sensing; TV, total variation. 
Several important approaches have been proposed to enhance angiographic reconstructions based on prior information (13-15). Methods that require high-quality prior acquisitions $(13,14)$ are not directly applicable to undersampled NCE MRA, where data are readily corrupted by aliasing and noise interference. Other methods exploiting temporal image correlations in dynamic acquisitions (15) may be inadequate for the static, high-spatial-resolution FIA targeted here.

Previous studies have also leveraged region-adaptive reconstructions to improve quality of angiograms (16-21). A group of studies have employed support detection for vascular masking in CE angiograms $(16,17)$. Vascular masking relies on heavily-suppressed static tissue in CE MRA, whereas bloodbackground contrast can be relatively impaired in NCE MRA. An alternative method is to utilize user-specified regions of interest (ROls) for support detection (18-20). However, such manual ROls can be broad and poorly localized to individual vessels. A recent study proposes in addition two-dimensional (2D) vessel segmentations to apply a spatially varying $\ell_{1}$ penalty (21). While this approach is promising, it does not consider the full threedimensional (3D) structure of vessels and finite-difference penalties, which may be critical for interference suppression.

Here we propose to attain high-contrast angiograms from undersampled data via a two-stage reconstruction. First, we generate $3 D$ vessel maps using a tractographic segmentation on Fourier reconstructions of undersampled NCE data (22). To dampen background signals, we then perform targeted reconstructions with spatially adaptive $\ell_{1}$-norm and total-variation penalties based on these maps. As demonstrated with simulations and in vivo experiments, the proposed strategy yields higher levels of background suppression compared with regular reconstructions, without compromising vessel depiction.

\section{METHODS}

In this work, we acquire peripheral angiograms in the extremities using a flow-independent technique and variable-density random sampling across $k$ space. We first obtain Fourier reconstructions of undersampled data following zero-filling and density compensation in $k$ space. We then leverage a powerful segmentation algorithm that jointly models tubular sections and branching structures to extract vasculature maps from these initial reconstructions. Finally, we perform targeted reconstructions, where these vasculature maps guide the enforcement of sparsity and total-variation constraints. The workflow of the proposed strategy is illustrated in Figure 1 and individual stages are described in detail in the following sections.

\section{Pulse sequence}

FIA of the peripheral extremities was acquired with a 3D magnetization-prepared pulse sequence $(3,5) . T_{2}$-prepared magnetization was captured with segmented, centric square-spiral phaseencode ordering (4). Each segment started with a linearly ramped series of RF excitations to minimize signal oscillations (23). Afterwards, fat-supressed data were acquired using an alternating repetition time SSFP sequence kernel (3). A recovery period was inserted between consecutive segments for magnetization recovery.

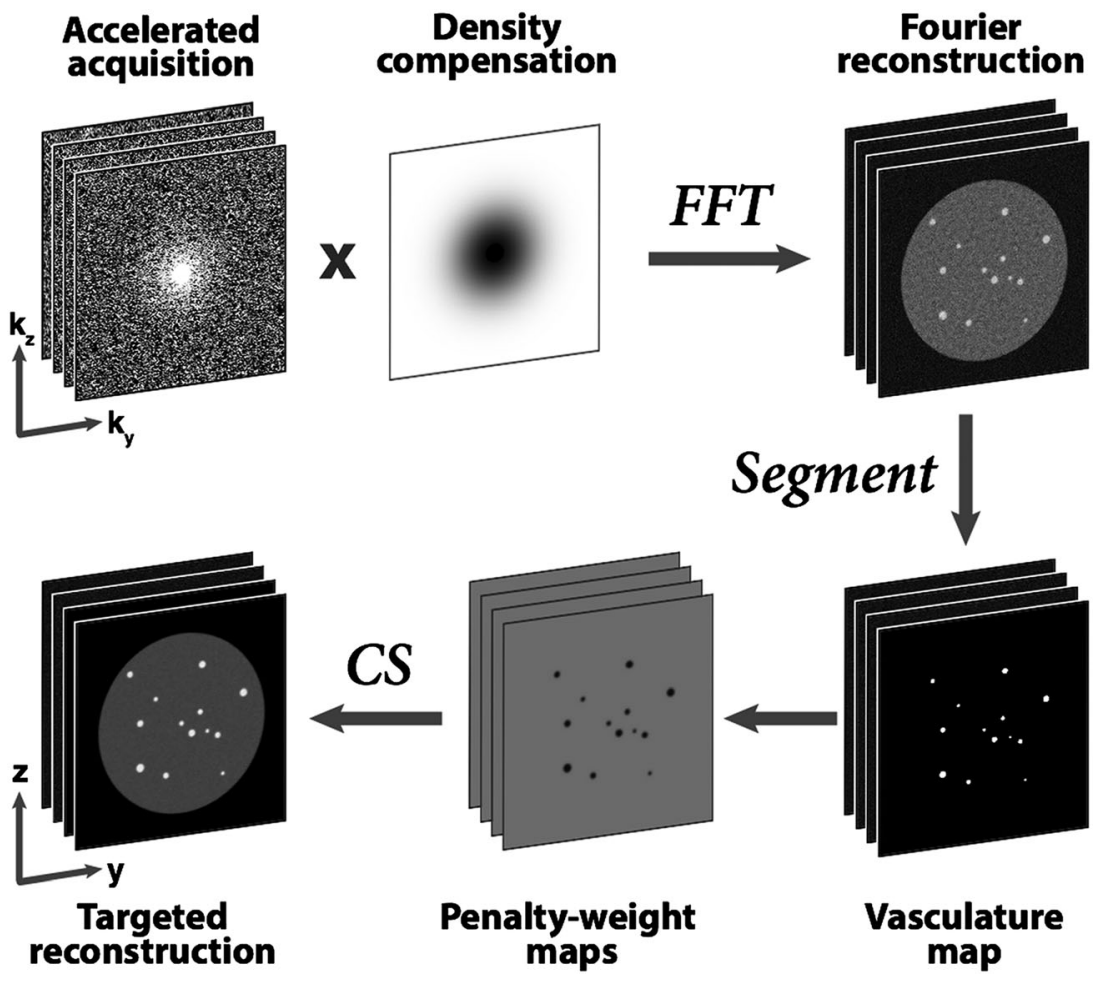

Figure 1. Proposed reconstruction strategy. Angiograms with variable-density undersampling in $k$ space are density-compensated and transformed to obtain Fourier reconstructions (ZF). A segmentation algorithm is then employed to trace vessel trees across the volume. In conventional CS, penalty terms are weighted uniformly across images. Here, penalty weights are selected based on segmented vasculature maps: smaller weights at vessel locations enabling targeted reconstructions. Note that data are not density-compensated during CS, but only to obtain ZF used for segmentation. 


\section{Sampling patterns}

To accelerate acquisitions, random sampling patterns were generated with a variable sampling density across $k$ space. Isotropic acceleration in two phase-encode dimensions was generated based on a polynomial density $(6,8,24)$ :

$$
P\left(k_{r}\right)=a_{1}\left(1-k_{r}\right)^{d}+a_{2}
$$

where $k_{r}$ is the $k$-space radius and $d$ and $a_{1,2}$ are constants that characterize the polynomial. Full sampling was utilized in the central $2 \%$ of $k$ space. For a given $d$, candidate sets of $a_{1,2}$ that yield the target acceleration factor $(R)$ were determined using a binary search algorithm. The resulting density for each set was used to generate 1000 random sampling patterns through a Monte Carlo simulation (6). Only patterns with a total number of samples within $1 \%$ of the ideal number (based on $R$ ) were accepted. The point-spread function (PSF) of each pattern was calculated by taking the inverse Fourier transform of the pattern and thus assuming an impulse object in the image domain. The level of aliasing energy was then taken as the magnitudesum of all pixels apart from the origin. The optimal sampling pattern was selected to attain minimal aliasing energy (see Supplementary Material: Table S1 and Fig. S1).

\section{Vasculature mapping}

Previous MRA studies have primarily employed vessel segmentations to enhance arterial-venous separation $(25,26)$ and to extract morphological features such as lumen size (27-29). Here we propose to use segmented vessel maps to enhance blood/background contrast in NCE MRA. We leverage a segmentation approach that we have demonstrated thoroughly for synthetic, coronary and cerebral angiograms (22,30,31). Our method jointly models branching structures with tubular sections by leveraging a fourth-order tensor model $(22,31)$. The tensor at each voxel in the vessel tree is constructed via nonnegative least-squares fitting performed on measurements of image gradient at 64 different orientations. This tensor is then decomposed into its singular vectors to identify major vessel tracts, including tubular sections as well as a variety of $n$-furcations such as $Y-, T-$, asymmetric- and crossing-junctions (22). Starting from a few seed points, this segmentation method can extract entire vessel trees in the extremities in less than 3 minutes (see Supplementary Table S2 and Fig. 2).

To extract vasculature maps, we first obtained zero-filled reconstructions of undersampled data. Data were compensated for variable $k$-space sampling density and zero-padded in three dimensions to double the $k$-space extent and minimize partial volume effects. To reduce noise levels, reconstructions were smoothed with a Gaussian kernel of length 7 and full width at half-maximum (FWHM) of 2.35. Afterwards, manual seed selection was performed to initiate the segmentation procedure. The seed points were selected on tubular sections of major vessels to avoid vessel junctions. The seeds were placed in vessels of high signal intensity located in superior or inferior cross-sections. The number of seeds prescribed for each anatomy depended on the number of disconnected vessel trees needing to be traced:
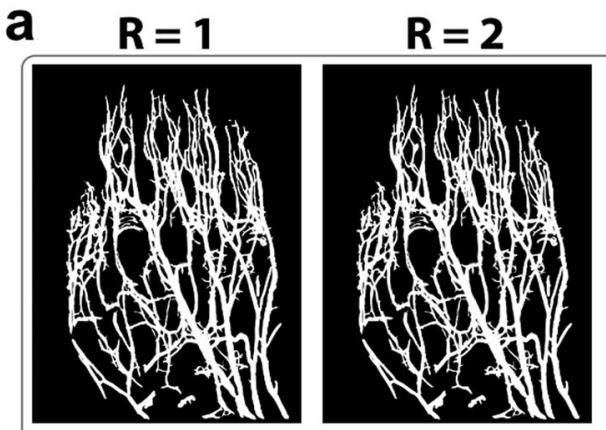

$\mathbf{R}=\mathbf{4}$

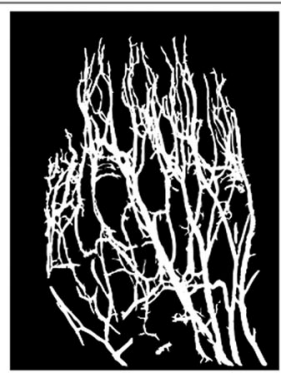

loss: $8.5 \%$
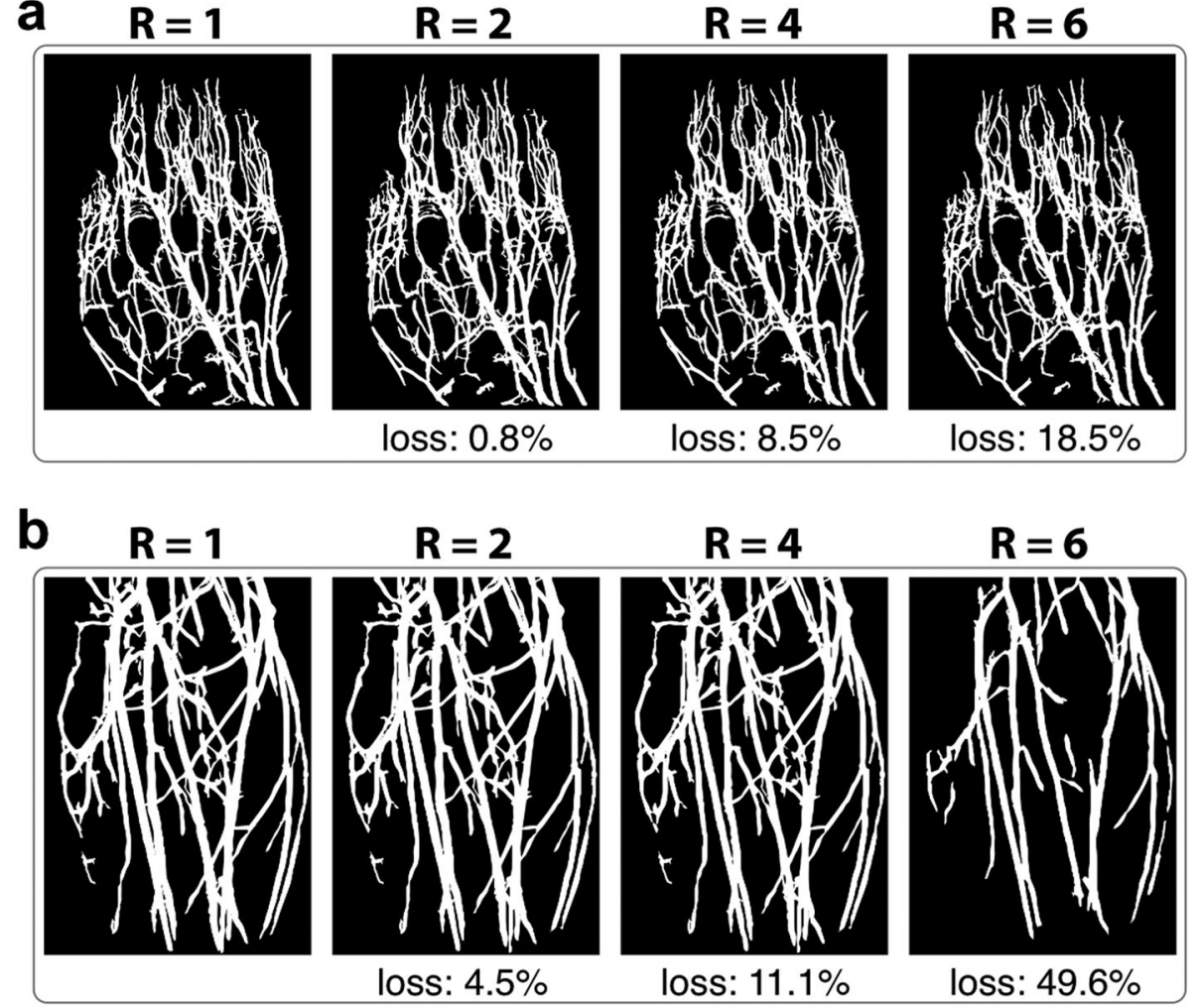

Figure 2. Vasculature maps segmented from undersampled angiograms at acceleration factors $R=1-6$. Vessel volumes for (a) hand angiograms and (b) lower leg angiograms are visualized with maximum-intensity projections (MIPs). Segmentation results at $R=1$ (fully sampled), 2 and 4 are visually similar to each other. For higher $R$, losses in vessel volume are apparent for particularly small vessels. The percentage volume loss in each map is listed with respect to the ideal map at $R=1$. 
seven seeds for hand, five seeds for lower leg and foot angiograms were selected inside major vessel branches. The seed points were identical across $R$. To demonstrate robustness against seed selection, segmentation was repeated five times with non-overlapping seeds (see Supplementary Fig. S2). Mismatch between the segmented vessel trees was less than $1.5 \%$ of the total vessel volume.

\section{Targeted compressed-sensing reconstructions}

Compressed sensing (CS) can estimate missing samples in MRI acquisitions when data have a compressible representation in a linear transform domain and sampling patterns yield incoherent aliasing in this domain (6). Nonlinear algorithms are then used during CS reconstructions to enforce compressibility and consistency with the acquired data. MRA datasets contain bright blood vessels surrounded by low-contrast background tissues. Therefore, CS is highly adept at reconstructing heavily undersampled angiograms (7-12).

\section{Optimization framework}

Here angiographic reconstructions are obtained via the following optimization $(6,8,24)$ :

$$
\min _{m}\left\|F_{u} m-Y\right\|_{2}^{2}+\left\|\lambda_{1} \circ m\right\|_{1}+\left\|\lambda_{2} \circ \Delta m\right\|_{1}
$$

where $m$ is the reconstructed image and the first term enforces consistency by minimizing the $\ell_{2}$-norm difference between the Fourier transform of the reconstruction $\left(F_{u} m\right)$ and the acquired data $(Y)$. Remaining are the penalty terms,

$$
\begin{aligned}
\left\|\lambda_{1} \circ m\right\|_{1} & =\sum_{i, j} \lambda_{1}(i, j)|m(i, j)| \\
\left\|\lambda_{2} \circ \Delta m\right\|_{1} & =\sum_{i, j} \lambda_{2}(i, j)\{|m(i+1, j)-m(i, j)| \\
& +|m(i, j+1)-m(i, j)|\}
\end{aligned}
$$

where $\circ$ is the Schur product, $\Delta$ is the summation of finitedifference transforms across cardinal dimensions and $i, j$ are the row and column indices of the image matrix. The $\ell_{1}$-norm penalty enforces sparse reconstructions in the image domain. The finite differences penalty, $T V(m)$ (i.e. total variation), enforces block-wise image homogeneity for denoising.

The problem in Equation [2] was solved using a nonlinear conjugate-gradient algorithm, implemented in MATLAB (Mathworks, Inc.). To compute the conjugate gradient of the $\ell_{1}$-norm, a fixed smoothing parameter of $10^{-15}$ was added during the absolute value calculation in all iterations. The stopping criterion was an improvement in the objective below a $0.1 \%$ threshold, which was observed to yield high-quality reconstructions in previous studies $(6,8,24)$. Reconstruction times are listed in Supplementary Table S2.

\section{Conventional weight selection}

In conventional $\mathrm{CS}, \lambda_{1,2}$ are uniform scalars across the entire image. Reconstructions were performed across a broad range of penalty weights, similar to previously considered ranges $(24,32)$. $\lambda_{1}$ was varied in the range [0 0.800] with a step size of 0.0005 in [0 0.040] and 0.020 in the remaining range. Meanwhile, $\lambda_{2}$ was varied with a step size of 0.0005 in the range [0 0.040]. $\lambda_{2}$ values greater than 0.040 caused undesirably high levels of block artefacts. The smallest pair of weights yielding sufficient artefact/noise suppression, without causing distortions or vessel loss, was determined by visual inspection and denoted as $\lambda_{1,2}=\lambda_{10,20}$ (see Table 1$)$.

\section{Targeted weight selection}

In angiograms, vessels appear as bright, small ellipsoidal structures in axial cross-sections, whereas background tissues appear as dark, broad regions (24). Larger $\lambda_{1}$ values promoting background sparsity will cause inadvertent loss of vessel signals. In addition, large $\lambda_{2}$ values promoting effective background denoising can yield suboptimal depiction of small vessels with limited contrast.

Here we propose to utilize spatially adaptive $\ell_{1}$-norm and TV penalties based on a spatial weight map derived from vessel segmentations. The binary segmentations indicate the location of vessels across the imaging volume. To improve robustness against segmentation errors and partial volume effects near the vessel boundaries, segmentations were dilated by one pixel in all dimensions and linearly ramped down from 1 to 0 across the dilated region. The maps were subtracted from 1 and normalized to calculate $W(i, j)$, which decreased from $r(r \geq 1)$ to 1 . This spatial map was then used to modify the penalty weights as follows:

$$
\begin{gathered}
\lambda_{1}(i, j) \rightarrow \lambda_{1} W(i, j) \\
\lambda_{2}(i, j) \rightarrow \lambda_{2} W(i, j) / r
\end{gathered}
$$

To improve background suppression, the $\ell_{1}$-norm penalty weights were scaled from $r \lambda_{1}$ in background regions to $\lambda_{1}$ in vessels. To minimize vessel loss, the TV penalty weights were scaled from $\lambda_{2}$ in background regions to $\lambda_{2} / r$ in vessels. The value of $r$ was selected to maximize image contrast without introducing significant image distortions.

\section{Phantom and in vivo reconstructions}

For each dataset, separate Fourier (ZF), conventional CS and targeted compressed sensing (TCS) reconstructions were computed using parameters listed in Table 1. For ZF, data were

Table 1. Reconstruction parameters for phantom and in vivo data at various acceleration factors $(R) . \lambda_{1}=10 \lambda_{10}$ for $\mathrm{CS}_{\text {high }}$, $\lambda_{1}=\lambda_{10}$, cont for $\mathrm{CS}_{\text {cont }}$ and $\lambda_{1}=\lambda_{10}$ for all remaining reconstructions. $\lambda_{2}=\lambda_{20}$ for all reconstructions. With this selection, TCS reconstructions scaled the $\ell_{1}$-norm penalty from $10 \lambda_{10}$ in the background to $\lambda_{10}$ in vessels and the TV penalty from $\lambda_{20}$ in the background to $\lambda_{20} / 10$ in vessels.

\begin{tabular}{|ccccccc|} 
& \multicolumn{3}{c}{ Phantom } & & \multicolumn{2}{c}{ In vivo } \\
\cline { 2 - 4 } \cline { 6 - 7 } & $\lambda_{10}$ & $\lambda_{20}$ & $\lambda_{10, \text { cont }}$ & & $\lambda_{10}$ & $\lambda_{20}$ \\
$\mathbf{1}$ & 0.032 & 0.032 & 0.520 & & 0.014 & 0.014 \\
$\mathbf{2}$ & 0.024 & 0.024 & 0.400 & & 0.010 & 0.010 \\
$\mathbf{4}$ & 0.016 & 0.016 & 0.340 & & 0.006 & 0.006 \\
$\mathbf{6}$ & 0.008 & 0.008 & 0.240 & & 0.001 & 0.002 \\
$\mathbf{8}$ & 0.004 & 0.004 & 0.120 & & 0.0005 & 0.001 \\
\hline
\end{tabular}


compensated for $k$-space sampling density and inverse Fouriertransformed. For CS, an identical $\lambda_{2}=\lambda_{20}$ was prescribed but several different $\lambda_{1}$ values were used. The first conservative CS was performed using $\lambda_{1}=\lambda_{10}\left(\mathrm{CS}_{\mathrm{low}}\right)$. A second heavier penalization was performed using $\lambda_{1}=10 \lambda_{10}$ ( $\left(\mathrm{CS}_{\text {high }}\right)$. For phantom datasets, a separate CS was calculated with an even larger $\lambda_{1}=\lambda_{10 \text {, cont }}\left(\mathrm{CS}_{\text {cont }}\right)$, where $\lambda_{\text {cont }}$ was selected to attain identical blood/background contrast to TCS. Because CS $_{\text {cont }}$ caused severe image artefacts, it was omitted in subsequent analyses.

TCS was performed using $\lambda_{1,2}=\lambda_{10,20}$ and $r \in\left[\begin{array}{ll}1 & 20\end{array}\right]$. For comparison, four other spatially adaptive CS methods were employed with the same parameters. First an intensity-weighted reconstruction was performed using weight maps derived from the intensity of ZF $\left(\mathrm{CS}_{\text {int }}\right)$. ZF reconstructions were normalized to a maximum amplitude of unity and then inverted to calculate $\mathrm{W}(i, j)$ similar to TCS. Secondly, iteratively reweighted $\mathrm{CS}\left(\mathrm{CS}_{\mathrm{IR}}\right)$ was performed (33). Weight maps for $\mathrm{CS}_{\mathrm{IR}}$ were updated at each iteration based on the reconstruction at the previous iteration. Unlike TCS or $\mathrm{CS}_{\mathrm{int}}, \mathrm{CS}_{\mathrm{IR}}$ maps did not reflect the region of signal support but rather the intensity of reconstructed images (33). Finally, two separate variants of the TCS method were implemented to assess the relative importance of using spatially adaptive weights on TV versus $\ell_{1}$ penalties. The first variant $\mathrm{TCS}_{n \mathrm{TV}}$ employed a spatially weighted $\ell_{1}$ and a fixed TV penalty, whereas the second variant $\mathrm{TCS}_{n \ell 1}$ employed a spatially weighted TV and a fixed $\ell_{1}$ penalty.

\section{Simulations}

To evaluate TCS independently from segmentation, we created two numerical phantoms that contained vessels immersed in a block of muscle tissue (Fig. $3 a$ and c).

Both tissues were modeled with circular cross-sections. The first phantom contained 25 vessels of diameters ranging from $0.33 \mathrm{~mm}$ ( 1 pixel) to $2 \mathrm{~mm}$ (6 pixels). The vessels were arranged on a $5 \times 5$ rectilinear grid within a muscle block of diameter $100 \mathrm{~mm}$ (300 pixels). The second phantom contained 13 vessels of diameters between 1.25 and $3.75 \mathrm{~mm}$, arranged randomly within the muscle block. Blood and muscle signals were simulated with the following parameters: $\alpha=60^{\circ}, \mathrm{TR}_{l, s}=3.45 /$ $1.15 \mathrm{~ms}, \mathrm{TE}=1.725 \mathrm{~ms}, T_{1} / T_{2}=1200 / 200 \mathrm{~ms}$ for blood (34) and $870 / 50 \mathrm{~ms}$ for muscle (35). The phantom images were sampled with a $384 \times 384$ grid over a $128 \times 128-\mathrm{mm}^{2}$ field of view. Circular cross-sections were created with a Fermi window using 1 pixel transition width. Finally, white Gaussian noise was added to yield a blood signal-to-noise ratio (SNR) of 20.

To examine penalty parameters used in TCS, we undersampled images of the first phantom with acceleration factors $R=1,2,4,6$ and 8 . For each $R, \lambda_{1,2}$ values were ranged between 0 and twice the $\lambda_{10,20}$ values listed in Table 1. Meanwhile, the ratio $(r)$ was varied in the range [1 20]. Larger TCS penalty weights yield improved background suppression (i.e. blood/muscle contrast), but cause distorted reconstructions of background signals. To assess reconstruction quality, a performance metric was calculated as the proportion of relative contrast difference to relative distortion level. Contrast improvement for each vessel was taken as

$$
\% \text { difference }=\frac{\text { Cont }_{T C S}-\text { Cont }_{Z F}}{\frac{1}{2}\left(\text { Cont }_{T C S}+\text { Cont }_{Z F}\right)} \times 100
$$

Distortion level was taken as the normalized dispersion index of background tissue,

$$
\Delta \mathrm{D}=\frac{D I_{T C S}}{D I_{Z F}}
$$

where $D I=\sigma^{2} / \mu$ and $\mu, \sigma$ denote the mean and standard deviation of the muscle signal, respectively.

TCS performance was assessed as a function of $r$ (Fig. 3b) when $\lambda_{1,2}=\lambda_{10,20}$. Performance increases rapidly as $r$ is initially raised above 1 and saturates for relatively large $r$. Note that higher $r$ enhances blood/background contrast at the expense of increased image distortions. Thus, for all $R, r=10$ was selected, which maintains more than $80 \%$ of the optimal performance. We then inspected the performance for $r=10, \lambda_{1}=n \lambda_{10}$ and $\lambda_{2}=n \lambda_{20}$, with $n$ varying in the range [ $\left[\begin{array}{ll}0 & 2\end{array}\right]$. Near-optimal performance is attained for $\lambda_{1,2}=\lambda_{10}, 20$. Independent optimizations of penalty weights indicate that $\lambda_{1,2}=\lambda_{10,20}$ and $r=10$ yield close to optimal performance for $\mathrm{CS}_{\text {int,IR }}$ as well. Therefore, they were prescribed for all reconstructions hereafter. To examine the effects of vessel size, TCS was calculated for 20 independent instances of additive noise. As expected, smaller vessels - more susceptible to signal loss - exhibit greater contrast improvement with TCS.

Next, we assessed the benefits of TCS on the second phantom, closely mimicking vessel sizes in the extremities (36). Blood/muscle contrast and spatial resolution were compared across CS and TCS (Fig. 3c). Spatial resolution was taken as the FWHM sizes of individual blood vessels normalized by the prescribed sizes in the numerical phantom. Statistical differences were assessed with Wilcoxon signed-rank tests.

To investigate robustness against segmentation errors, separate TCS reconstructions were performed by simulating losses in segmented vessel maps (Fig. 3d). The ideal vessel masks were eroded to yield a volumetric loss varying between 0 and $30 \%$ of the total vessel volume. The erosion process used a random voxel selection that maintained spatial contiguity for each vessel. TCS was calculated for 20 distinct instances of vessel erosion and additive noise. Blood/muscle contrast and normalized vessel sizes were measured for each vessel individually and then averaged across 13 vessels.

\section{Experiments}

To demonstrate TCS, we first acquired in vivo hand and lower-leg angiograms on a $1.5 \mathrm{~T}$ GE Signa EX scanner with CV/i gradients (maximum strength of $40 \mathrm{mT} / \mathrm{m}$ and slew rate of $150 \mathrm{~T} / \mathrm{m} / \mathrm{s}$ ). Angiographic acquisitions were performed using a 3D magnetization-prepared SSFP pulse sequence $(3,5)$. High-resolution hand angiograms were collected in a healthy subject (male, age 27) using an eight-channel receive-only knee array with the following parameters: $0.5 \times 0.5 \times 0.5 \mathrm{~mm}^{3}$ spatial resolution, $320 \times 240 \times 120$ encoding matrix, $\alpha=60^{\circ}, \mathrm{TR}_{l, s}=3.6 / 1.2 \mathrm{~ms}, \mathrm{TE}=1.8 \mathrm{~ms}, 125 \mathrm{kHz}$ readout bandwidth, $80 \mathrm{~ms} T_{2}$ preparation, 10-tip linear ramp catalyzation, $10 \mathrm{k}$-space segments, 4-s intersegment recovery time and a total scan time of $3 \mathrm{~min} 40 \mathrm{~s}$. Data from individual coils were sum-of-squares combined (37). Hand angiograms were retrospectively undersampled by acceleration factors of $R=1$ (fully sampled), 2, 4, 6 and 8 . Meanwhile, prospectively undersampled lower-leg angiograms were collected in a healthy subject (female, age 28) using a transmit-receive quadrature extremity coil and with identical parameters to the hand protocol except for $1 \times 1 \times 1 \mathrm{~mm}^{3}$ spatial resolution, $192 \times 128 \times 128$ encoding matrix, $\mathrm{TR}_{l, s}=3.45 / 1.15 \mathrm{~ms}, \mathrm{TE}=1.725 \mathrm{~ms}$. Separate acquisitions were performed at $R=(1,2,4,6,8)$ with number of magnetization preparations $N=(4,16,22,24,26)$ respectively; scan time for each acquisition was $1 \mathrm{~min} 30 \mathrm{sec}$. Vasculature maps were extracted from ZF reconstructions of undersampled data. 


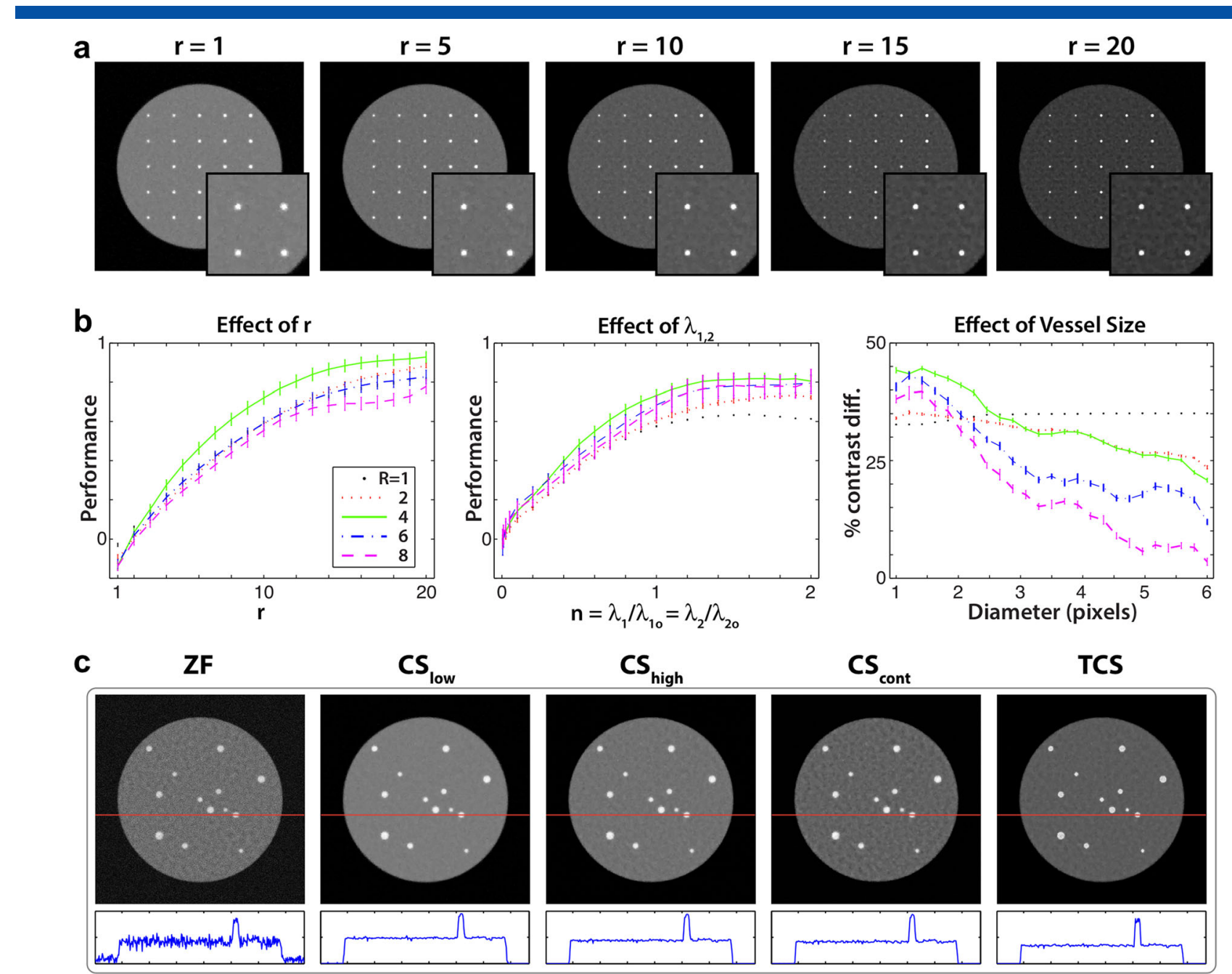

c

d
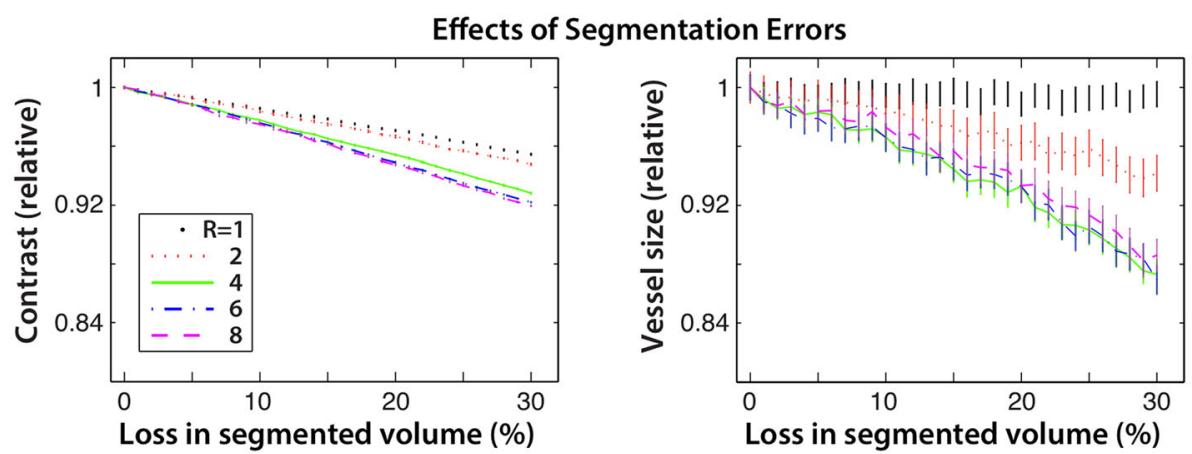

Figure 3. (a) A phantom with 25 blood vessels of sizes $0.33-2 \mathrm{~mm}$ enclosed by muscle. Targeted CS (TCS) reconstructions were calculated using $r \in\left[\begin{array}{ll}12 & 20\end{array}, \lambda_{1,2}=\lambda_{10}, 20\right.$ and $R=1-8$. Results are shown for $R=1$. Overlaid boxes show the magnified lower-right portions of phantom images. Higher $r$ values enhance blood/muscle contrast, but image distortions become prominent for $r \geq 15$. (b) TCS performance, taken as the ratio of contrast improvement to dispersion level (mean \pm sem -standard error- across vessels). For all $R, r=10$ and $\lambda_{1,2}=\lambda_{10}$, 20 yields close to optimal reconstruction performance (left and middle panels). TCS using $\lambda_{1,2}=\lambda_{10}, 20$ and $r=10$ was repeated for 20 random instances of additive image noise. Contrast improvement is plotted for each vessel diameter (right panel; mean \pm sem across 20 images). The improvement in contrast is greater for smaller vessels. (c) A separate phantom with 13 blood vessels of sizes $1.25-3.75 \mathrm{~mm}$. Fourier reconstructions (ZF), conventional $C S\left(\mathrm{CS}_{\text {low, }} \mathrm{CS}_{\text {high, }} \mathrm{CS}_{\text {cont }}\right)$ and TCS were calculated for $R=4$. The panel below each image shows a sample line profile (across the red line). TCS improves blood/muscle contrast compared with $\mathrm{CS}_{\text {low }}$ high, meanwhile $\mathrm{CS}_{\text {cont }}$ causes significant image distortions. (d) TCS reconstructions were obtained, while losses in segmented vessel maps were simulated by random erosions of the ideal map. Contrast and vessel sizes were measured relative to a TCS reconstruction based on the ideal map (mean \pm sem across 20 instances of erosion).

To validate TCS results in a broader population, we next collected lower-leg angiograms from four healthy subjects (one female, three males; ages 27-32) and foot angiograms from four healthy subjects (one female, three males; ages 27-32). Data were acquired on a 1.5 T GE Signa EX scanner using a quadrature extremity coil, with identical parameters to the lower-leg protocol listed above. The only exception was a fixed number of magnetization preparations $N=4$ for all accelerations. Vessel maps 
Table 2. Contrast and resolution in simulated data. Contrast: average blood/muscle contrast on phantom data at various $R$. Raw contrast values are listed, together with the percentage difference in contrast between each method and ZF. Resolution: relative radius of blood vessels in phantom images (mean \pm s.d. across 13 vessels) compared with the actual vessel sizes

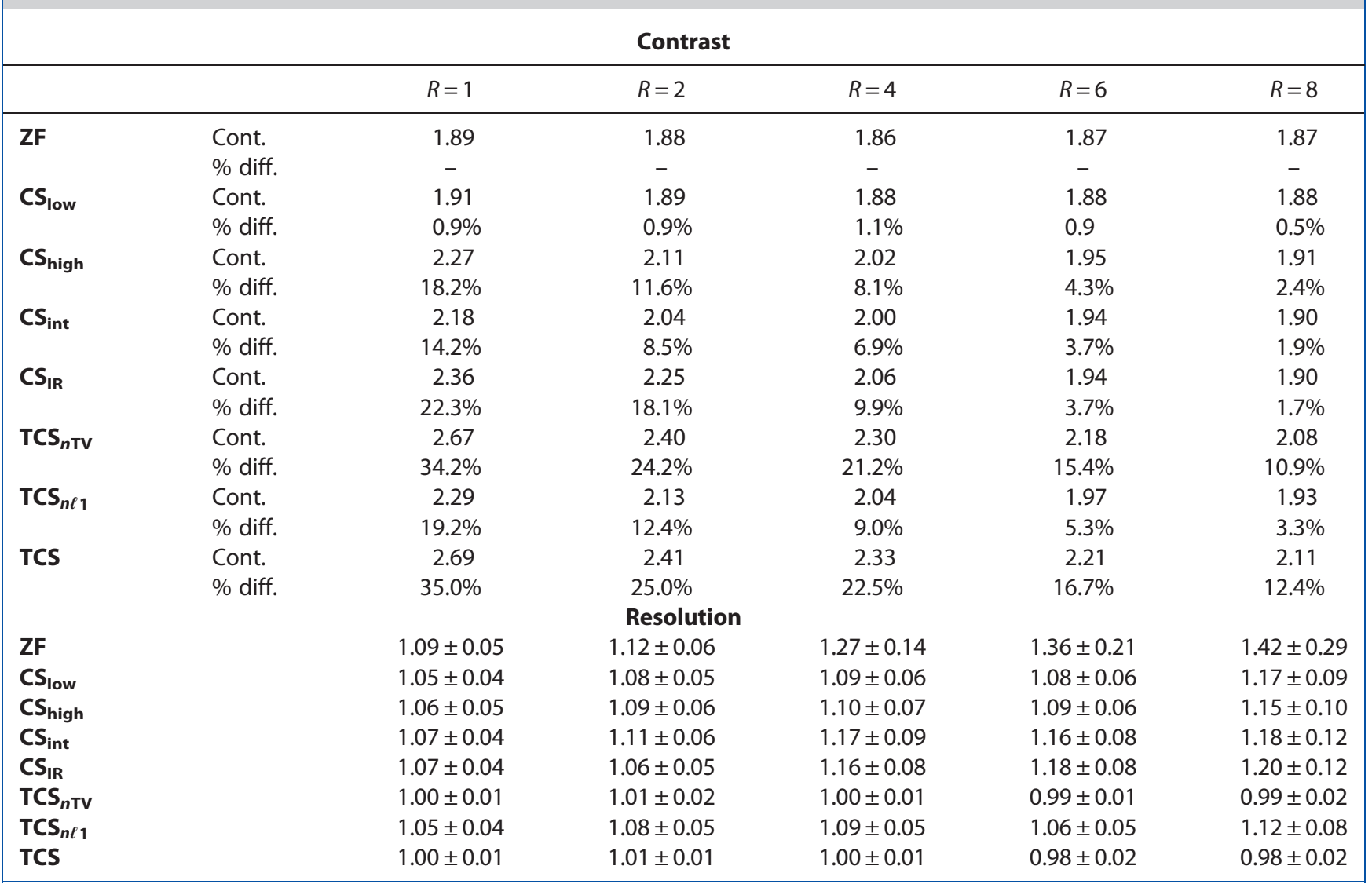

from fully-sampled data were used to demonstrate effects of spatially-adaptive penalization.

CS and TCS reconstructions were then calculated on 2D crosssections. Reconstruction parameters were selected by examining TCS performance as a function of $r$ and $\lambda_{1,2}$. It was observed that near-optimal performance is obtained for $r=10$ and $\lambda_{1,2}=\lambda_{10,20}$. To minimize partial volume effects in maximum-intensity projection (MIP) views, all reconstructed datasets were upsampled by a factor of two in all dimensions by zero-padding in $k$ space.

To assess image contrast, average blood and muscle signals were measured in 13 coronal cross-sections spanning across the entire volume. Within a single section, two separate regions of interest (ROIs) with homogeneous blood and muscle signal were selected. Signals were averaged within these ROls and the ratio of blood to muscle signal was taken as the contrast for each cross-section. ROIs were identical across reconstructions of the same anatomy. In hand angiograms, blood signal was measured on superficial to deep segments of digital radial and ulnar arteries (569 \pm 142 voxels, mean \pm s.d. across 13 cross-sections), while muscle signal was measured in the palmar region $(473 \pm 68$ voxels). In lower-leg angiograms, blood signal was measured on proximal to distal segments of the tibial and peroneal arteries ( $73 \pm 20$ voxels), while muscle signal was measured across neighboring tissue $(101 \pm 20)$. In foot angiograms, blood signal was measured on dorsal metatarsal and plantar arteries ( $52 \pm 34$ voxels) and muscle signal was measured across neighboring tissue $(80 \pm 29)$.
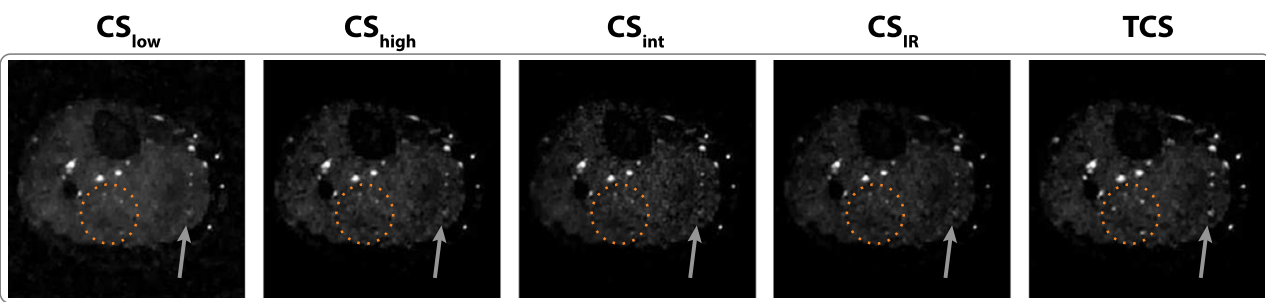

Figure 4. Lower-leg angiograms reconstructed using conventional CS with uniformly weighted penalty terms ( $\left(\mathrm{CS}_{\text {low, }} \mathrm{CS}_{\text {high }}\right)$, $C S$ with spatially weighted penalty terms based on intensity of ZF reconstructions $\left(\mathrm{CS}_{\text {int }}\right)$, iteratively reweighted $\mathrm{CS}\left(\mathrm{CS}_{\mathrm{IR}}\right)$ and TCS. Representative axial sections are shown for $R=4$. $\mathrm{CS}_{\text {high }}, \mathrm{CS}_{\text {int }}$ and $\mathrm{CS}_{\mathrm{IR}}$ suffer from signal losses, particularly in relatively small or low-intensity vessels. In contrast, TCS improves background suppression while preserving detailed depiction of vasculature (marked with ellipses and arrows). 

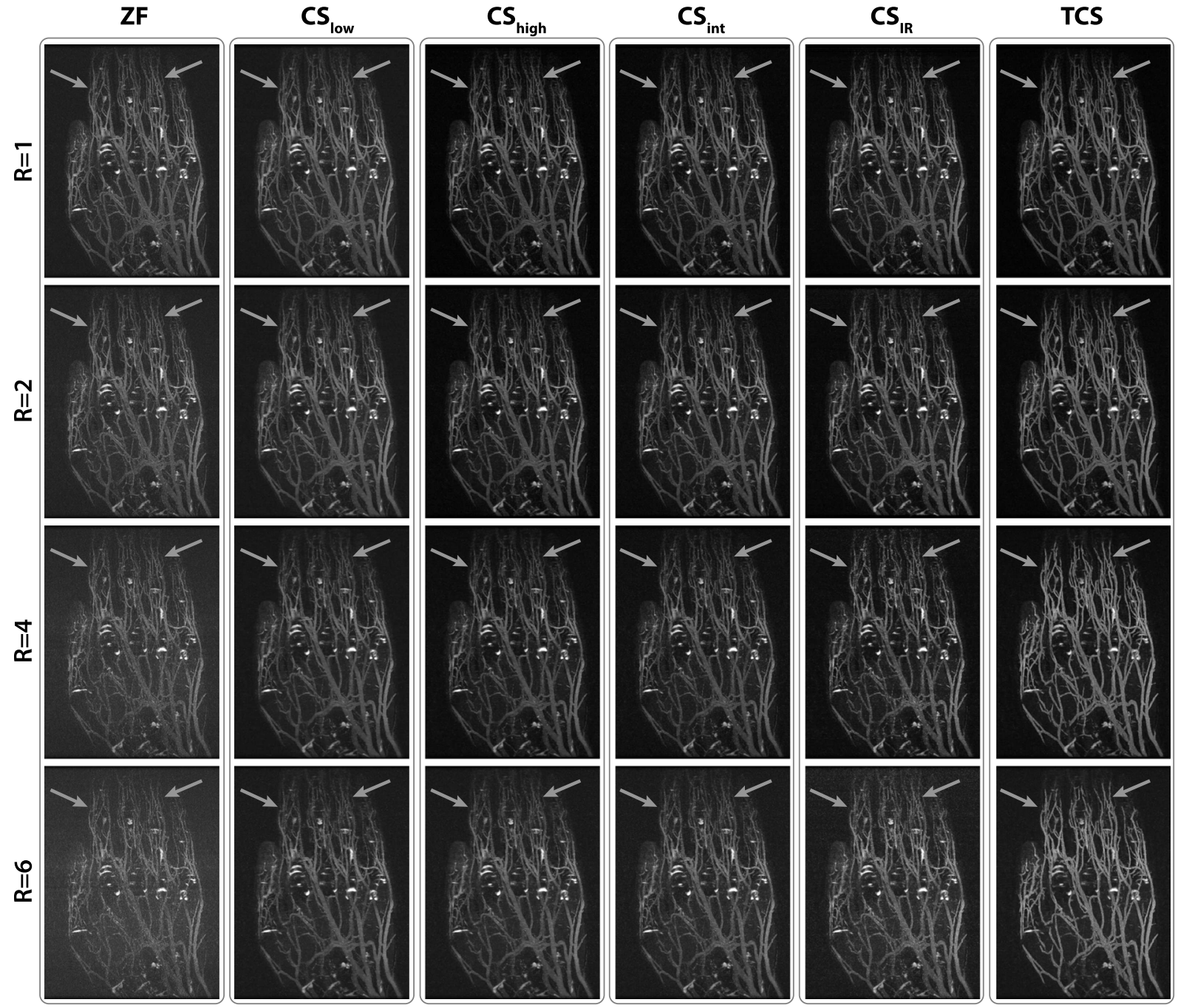

Figure 5. MIPs of hand angiograms reconstructed with $\mathrm{ZF}, \mathrm{CS}$ and TCS, for $R=1-6 . \mathrm{CS}_{\mathrm{IR}}$ suffers increasingly from loss of small vessels for higher $R$. Furthermore, bright synovial fluid causes suboptimal vessel contrast in ZF and $\mathrm{CS}_{\mathbb{I R}}$. In contrast, TCS alleviates vessel loss while improving suppression of background signals (marked with arrows).

To examine potential vessel-thickening artefacts that may be introduced during segmentation and reconstruction steps, two control analyses were performed in hand and lower-leg datasets. First, the set of non-overlapping voxels in segmented maps was examined across $R$. At each $R$, the number of additional voxels beyond those included in $R=1$ was counted. A percentage-increase metric was taken as the ratio of this number to the total number of voxels at $R=1$ and it was used to quantify thickening in vessel maps. Secondly, vessel thickness was measured on reconstructed angiograms. For this analysis, vessels of various sizes were selected across 10 different axial cross-sections. The thickness of each vessel was taken as the diameter of the FWHM region, which ranged from 1 to $4 \mathrm{~mm}$. The level of blurring in each reconstruction method was calculated as the relative vessel diameter compared with a ZF reconstruction of fully sampled data $(R=1)$.

Two expert radiologists evaluated the diagnostic quality of reconstructed images by consensus. At each $R$, MIP views were used to compare images from different reconstruction methods (without method identifiers). Image contrast, vessel demarcation and distalbranch visualization in each image were rated separately using a five-point scale (5 excellent, 4 good, 3 moderate, 2 limited, 1 poor).
Statistical differences in all quantitative results were assessed with Wilcoxon signed-rank tests.

\section{RESULTS}

Blood/muscle contrast and resolution on simulated phantom images are listed in Table 2. At each $R$, TCS improves contrast significantly compared with all other methods including $\mathrm{TCS}_{n \mathrm{TV}}$ and $\mathrm{TCS}_{n \ell 1}(P<0.05)$. We find an improvement of $21.5 \pm 8.7 \%$ over $\mathrm{CS}_{\text {low }}$ (mean \pm s.d. across $R$ ) and $11.3 \pm 2.8 \%$ over $\mathrm{CS}_{\mathrm{IR}_{\mathrm{R}}}$, the closest CS competitor to TCS. The contrast improvement is greater for lower $R$ values, where heavier sparsity penalties can be enforced due to increased acquisition SNR. Furthermore, TCS maintains improved spatial resolution compared with other CS methods and $\mathrm{TCS}_{n \ell 1}$ at each $R(P<0.05)$. This improvement in resolution is more prominent in higher $R$ datasets, which are more susceptible to resolution loss.

To assess the reliability of TCS against segmentation errors, phantom images were reconstructed for varying volumetric losses in vessel maps. TCS using randomly eroded versions of the ideal vessel map was compared with TCS using the ideal 

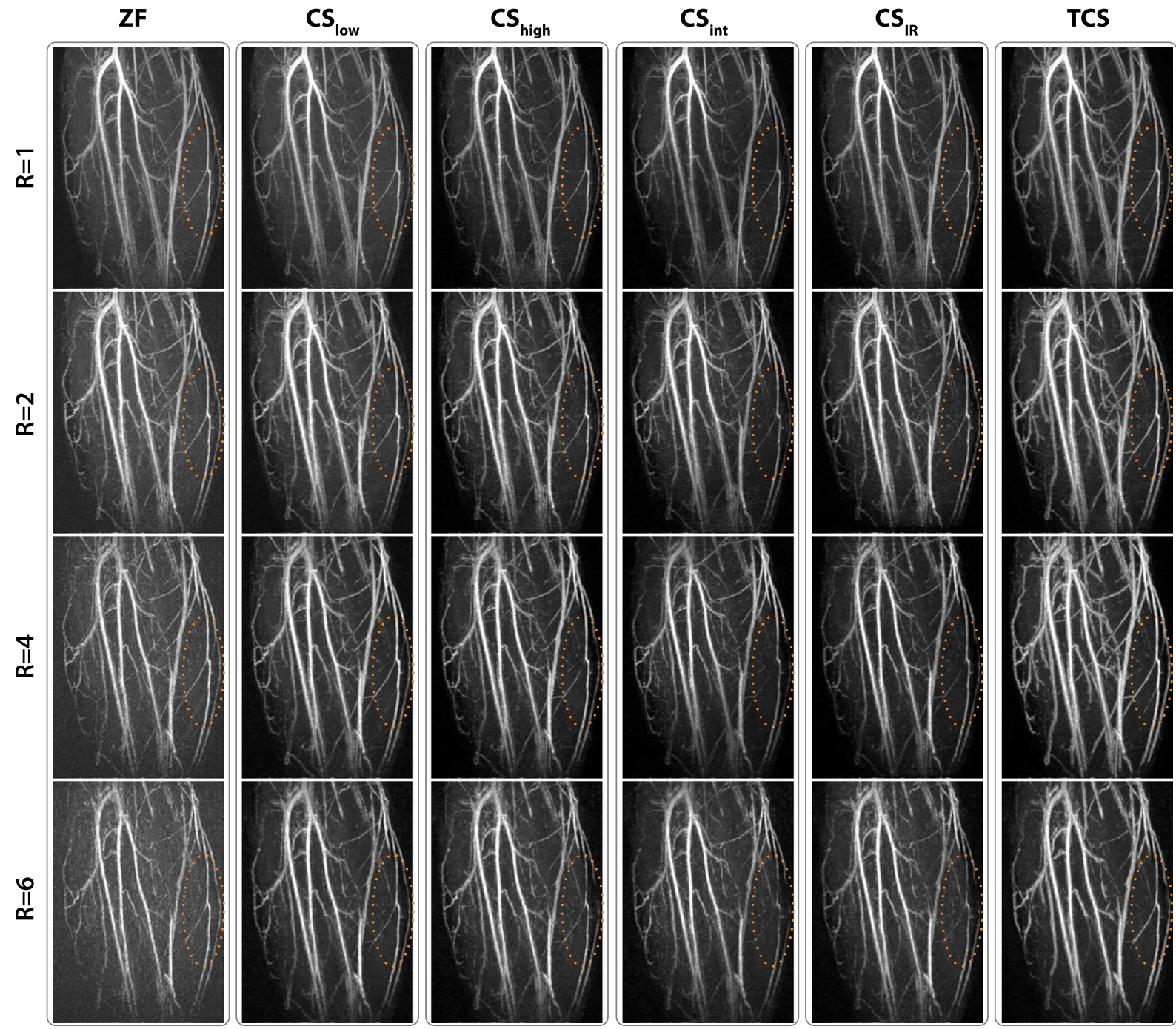

Figure 6. MIPs of lower leg angiograms reconstructed with ZF, CS and TCS, for $R=1-6$. There is visible loss of low-intensity and small vessels in CS $\mathrm{IR}_{\text {. }}$ TCS achieves improved blood/muscle contrast with no visible vessel loss up to $R=4$ (marked with ellipses). Due to reduction of segmented volumes for $R=6$ (Fig. 2), some small vessels are depicted suboptimally.

map (Fig. 3d). At all $R$ values, contrast remains within $8 \%$ and vessel size remains within $12 \%$ of the ideal values for up to $30 \%$ segmentation loss. These results indicate that TCS shows considerable performance in the presence of moderate segmentation errors.

Representative reconstructions of in vivo hand and lower-leg angiograms are shown in Figures 4-6. TCS visibly improves blood/background contrast and enhances vessel depiction via tailored penalty weights. Our control analyses indicate that the segmented vessel maps at high $R$ values (shown in Fig. 2) do not exhibit any thickening compared with the ideal maps at $R=1$. Furthermore, we find no significant differences in vessel thickness across reconstruction methods and across $R$ $(P>0.125$; see Supplementary Table S3). Thus the prominent appearance of vessel trees in TCS reconstructions is due to improved angiographic contrast. While some small vessels are less effectively visualized at $R \geq 6$, due to reduction of segmented volumes (Fig. 2), TCS depicts major vessels reliably, including the digital-radial/ulnar arteries in the hand and popliteal/peroneal arteries in the lower leg. Blood/background contrast measurements in representative hand and lower-leg angiograms are listed in Table 3. At each $R$, TCS yields significantly higher contrast than all other methods including $\mathrm{TCS}_{n \mathrm{TV}}$ and $\mathrm{TCS}_{n \ell 1}$ $(P<0.05)$. In the hand, the improvement is $71.3 \pm 28.9 \%$ over $\mathrm{CS}_{\text {low }}$ and $33.0 \pm 6.6 \%$ over $\mathrm{CS}_{\mathrm{IR}}$. In the lower leg, the improvement is $38.5 \pm 8.5 \%$ over $\mathrm{CS}_{\text {low }}$ and $22.1 \pm 6.6 \%$ over $\mathrm{CS}_{\mathrm{IR}}$.

The contrast measurements in lower-leg and foot angiograms collected in a broader population of subjects are listed in Table 4. Across subjects, TCS achieves higher contrast than all other reconstructions at each $R(P<0.05)$. In the lower leg, the improvement is $30.6 \pm 11.3 \%$ over $\mathrm{CS}_{\text {low, }} 14.9 \pm 1.8 \%$ over $\mathrm{CS}_{\mathrm{IR}}$, $3.0 \pm 1.4 \%$ over $\mathrm{TCS}_{n \mathrm{TV}}$ and $13.8 \pm 2.7 \%$ over $\mathrm{TCS}_{n \ell 1}$. In the foot, the improvement is $28.1 \pm 7.0 \%$ over $\mathrm{CS}_{\text {low, }} 10.4 \pm 4.8 \%$ over $\mathrm{CS}_{\mathrm{IR}}$, $3.0 \pm 1.5 \%$ over $\mathrm{TCS}_{n \mathrm{TV}}$ and $11.4 \pm 2.6 \%$ over $\mathrm{TCS}_{n \ell 1}$. Consistent with simulation results, contrast improvement for in vivo data is greater for lower $R$.

Radiological assessments of image contrast, vessel demarcation and distal-branch visualization concur that the proposed method enhances image quality (see Supplementary Table S4). Across all subjects, TCS achieves higher image contrast and vessel demarcation scores than all other CS reconstructions at each $R(P<0.05)$, except for $R=1$, where we find no significant 
Table 3. Contrast: representative single-subject data. Blood/muscle contrast (mean \pm s.d. across 13 sections) in hand and lower leg angiograms at various $R$. Raw contrast values are listed, together with the percentage difference in contrast between each method and ZF

Hand Data

\begin{tabular}{|c|c|c|c|c|c|c|}
\hline & & $R=1$ & $R=2$ & $R=4$ & $R=6$ & $R=8$ \\
\hline \multirow[t]{2}{*}{ ZF } & Cont. & $2.32 \pm 0.10$ & $2.25 \pm 0.10$ & $2.06 \pm 0.12$ & $2.02 \pm 0.13$ & $2.01 \pm 0.12$ \\
\hline & $\%$ diff. & - & - & - & - & - \\
\hline \multirow[t]{2}{*}{$\mathrm{CS}_{\text {low }}$} & Cont. & $2.35 \pm 0.11$ & $2.23 \pm 0.11$ & $2.04 \pm 0.09$ & $1.97 \pm 0.10$ & $2.00 \pm 0.08$ \\
\hline & $\%$ diff. & $1.35 \%$ & $-1.14 \%$ & $-0.82 \%$ & $-2.86 \%$ & $-0.11 \%$ \\
\hline \multirow[t]{2}{*}{$\mathrm{CS}_{\text {high }}$} & Cont. & $6.92 \pm 0.87$ & $3.55 \pm 0.29$ & $2.62 \pm 0.14$ & $2.08 \pm 0.12$ & $2.11 \pm 0.09$ \\
\hline & $\%$ diff. & $99.63 \%$ & $44.83 \%$ & $23.79 \%$ & $2.96 \%$ & $5.06 \%$ \\
\hline \multirow[t]{2}{*}{$\mathrm{CS}_{\text {int }}$} & Cont. & $5.50 \pm 0.5$ & $3.45 \pm 0.27$ & $2.70 \pm 0.14$ & $2.17 \pm 0.12$ & $2.18 \pm 0.09$ \\
\hline & $\%$ diff. & $81.51 \%$ & $42.12 \%$ & $26.97 \%$ & $6.83 \%$ & $8.58 \%$ \\
\hline \multirow[t]{2}{*}{$\mathrm{CS}_{\mathrm{IR}}$} & Cont. & $5.63 \pm 0.65$ & $3.71 \pm 0.31$ & $3.03 \pm 0.16$ & $2.38 \pm 0.15$ & $2.41 \pm 0.11$ \\
\hline & $\%$ diff. & $83.38 \%$ & $49.08 \%$ & $38.00 \%$ & $16.12 \%$ & $18.28 \%$ \\
\hline \multirow[t]{2}{*}{$\mathrm{TCS}_{n \mathrm{TV}}$} & Cont. & $8.56 \pm 1.03$ & $4.80 \pm 0.37$ & $4.03 \pm 0.21$ & $2.86 \pm 0.18$ & $2.91 \pm 0.13$ \\
\hline & $\%$ diff. & $114.79 \%$ & $72.30 \%$ & $64.72 \%$ & $34.34 \%$ & $36.69 \%$ \\
\hline \multirow[t]{2}{*}{$\operatorname{TCS}_{n \ell 1}$} & Cont. & $7.19 \pm 0.87$ & $3.73 \pm 0.31$ & $2.87 \pm 0.15$ & $2.36 \pm 0.13$ & $2.40 \pm 0.10$ \\
\hline & $\%$ diff. & $102.56 \%$ & $49.55 \%$ & $32.73 \%$ & $15.22 \%$ & $17.92 \%$ \\
\hline \multirow[t]{2}{*}{ TCS } & Cont. & $8.70 \pm 1.04$ & $4.99 \pm 0.38$ & $4.30 \pm 0.22$ & $3.16 \pm 0.21$ & $3.22 \pm 0.15$ \\
\hline & $\%$ diff. & $115.89 \%$ & $75.64 \%$ & $70.51 \%$ & $44.05 \%$ & $46.50 \%$ \\
\hline \multicolumn{7}{|c|}{ Lower Leg Data } \\
\hline \multirow[t]{2}{*}{ ZF } & Cont. & $1.82 \pm 0.24$ & $2.11 \pm 0.27$ & $2.24 \pm 0.14$ & $2.61 \pm 0.18$ & $2.74 \pm 0.20$ \\
\hline & $\%$ diff. & - & - & - & - & - \\
\hline \multirow[t]{2}{*}{$\mathrm{CS}_{\text {low }}$} & Cont. & $1.84 \pm 0.27$ & $2.13 \pm 0.32$ & $2.31 \pm 0.20$ & $2.66 \pm 0.20$ & $2.72 \pm 0.32$ \\
\hline & $\%$ diff. & $1.06 \%$ & $1.08 \%$ & $2.95 \%$ & $2.06 \%$ & $-0.49 \%$ \\
\hline \multirow[t]{2}{*}{$\mathrm{CS}_{\text {high }}$} & Cont. & $2.52 \pm 0.59$ & $2.68 \pm 0.57$ & $2.71 \pm 0.31$ & $2.79 \pm 0.24$ & $2.81 \pm 0.41$ \\
\hline & $\%$ diff. & $31.90 \%$ & $23.83 \%$ & $19.18 \%$ & $6.67 \%$ & $2.79 \%$ \\
\hline \multirow[t]{2}{*}{$\mathrm{CS}_{\text {int }}$} & Cont. & $2.46 \pm 0.48$ & $2.69 \pm 0.55$ & $2.79 \pm 0.30$ & $2.85 \pm 0.20$ & $2.85 \pm 0.28$ \\
\hline & $\%$ diff. & $29.66 \%$ & $24.36 \%$ & $22.05 \%$ & $8.91 \%$ & $4.19 \%$ \\
\hline \multirow[t]{2}{*}{$\mathrm{CS}_{\mathrm{IR}}$} & Cont. & $2.50 \pm 0.58$ & $2.74 \pm 0.59$ & $2.77 \pm 0.36$ & $2.87 \pm 0.28$ & $2.79 \pm 0.45$ \\
\hline & $\%$ diff. & $31.24 \%$ & $26.22 \%$ & $21.29 \%$ & $9.47 \%$ & $1.80 \%$ \\
\hline \multirow[t]{2}{*}{$\mathrm{TCS}_{n \mathrm{TV}}$} & Cont. & $3.04 \pm 0.69$ & $3.11 \pm 0.65$ & $3.28 \pm 0.39$ & $3.30 \pm 0.31$ & $3.57 \pm 0.50$ \\
\hline & $\%$ diff. & $50.03 \%$ & $38.46 \%$ & $37.84 \%$ & $23.37 \%$ & $26.51 \%$ \\
\hline \multirow[t]{2}{*}{$\operatorname{TCS}_{n \ell 1}$} & Cont. & $2.57 \pm 0.60$ & $2.76 \pm 0.58$ & $2.84 \pm 0.32$ & $2.99 \pm 0.27$ & $3.10 \pm 0.45$ \\
\hline & $\%$ diff. & $34.11 \%$ & $26.72 \%$ & $23.70 \%$ & $13.65 \%$ & $12.58 \%$ \\
\hline \multirow[t]{2}{*}{ TCS } & Cont. & $3.11 \pm 0.70$ & $3.17 \pm 0.65$ & $3.42 \pm 0.40$ & $3.52 \pm 0.34$ & $3.88 \pm 0.54$ \\
\hline & $\%$ diff. & $52.08 \%$ & $40.28 \%$ & $41.62 \%$ & $29.76 \%$ & $34.63 \%$ \\
\hline
\end{tabular}

difference. While comparisons are less uniform for distal-branch visualization, the average visualization score across $R$ is higher for TCS compared with all other reconstructions $(P<0.05)$.

\section{DISCUSSION}

Here we propose a reconstruction strategy (TCS) for NCE angiograms that leverages vasculature maps extracted from undersampled data, without relying on prior information. The morphological information in these maps is used to apply order-of-magnitude heavier sparsity and TV penalties across background tissues compared with vessels. As such, TCS enhances blood/background contrast compared with conventional CS without degrading vessel depiction.

A recent study has used $2 \mathrm{D}$ segmentations to apply a spatially varying $\ell_{1}$ penalty (21). While this previous approach has similar motivations to TCS, our study differs in several important aspects. Firstly, we use a tractographic segmentation to exploit $3 \mathrm{D}$ structure and leverage vessel contiguity in the superior- inferior direction. Secondly, we utilize concurrent spatial weighting on both $\ell_{1}$-norm and TV penalties to minimize vessel signal loss. Our results show that concurrent weighting in TCS enhances image quality over weighting either term alone. Lastly, apart from noise/aliasing reduction aimed previously, here we demonstrate contrast enhancement that improves vessel depiction in contrast-limited NCE MRA significantly.

The practical benefits of TCS depend on the coverage of the segmented vasculature maps. Our simulations suggest that TCS maintains considerable performance with up to $30 \%$ volume loss in segmented maps. However, with increased aliasing at high $R$, small vessels with low contrast may be missed and thereby incur heavy penalties during TCS. Here, some small, low-contrast branches were not segmented at $R=6$ and 8; loss of high-spatial-frequency information in TCS became prominent at $R=8$ (not shown). Such losses may mimic stenoses in minor vessel branches. To minimize misassessment, segmented maps can be dilated more broadly and reconstruction penalties may be limited at higher $R$. Alternatively, segmentation and reconstruction stages can be cast as a joint optimization problem (38), 
Table 4. Contrast: population data. Blood/muscle contrast (mean \pm s.d. across 4 subjects) in lower-leg and foot angiograms at various $R$. Raw contrast values are listed, together with the percentage difference in contrast between each method and ZF

\begin{tabular}{|c|c|c|c|c|c|c|}
\hline \multicolumn{7}{|c|}{ Lower Leg Data } \\
\hline & & $R=1$ & $R=2$ & $R=4$ & $R=6$ & $R=8$ \\
\hline \multirow[t]{2}{*}{ ZF } & Cont. & $2.19 \pm 0.28$ & $2.17 \pm 0.28$ & $2.12 \pm 0.27$ & $2.08 \pm 0.25$ & $2.04 \pm 0.25$ \\
\hline & $\%$ diff. & - & - & - & - & - \\
\hline \multirow[t]{2}{*}{$\mathrm{CS}_{\text {low }}$} & Cont. & $2.21 \pm 0.29$ & $2.17 \pm 0.30$ & $2.10 \pm 0.30$ & $2.05 \pm 0.29$ & $2.02 \pm 0.31$ \\
\hline & \% diff. & $1.2 \pm 0.8 \%$ & $-0.1 \pm 1.3 \%$ & $-1.1 \pm 2.0 \%$ & $-1.9 \pm 2.9 \%$ & $-1.4 \pm 4.1 \%$ \\
\hline \multirow[t]{2}{*}{$\mathrm{CS}_{\text {high }}$} & Cont. & $2.99 \pm 0.43$ & $2.68 \pm 0.38$ & $2.39 \pm 0.37$ & $2.12 \pm 0.31$ & $2.07 \pm 0.31$ \\
\hline & \% diff. & $30.8 \pm 6.2 \%$ & $20.7 \pm 3.5 \%$ & $11.6 \pm 3.2 \%$ & $1.7 \pm 3.4 \%$ & $1.3 \pm 3.9 \%$ \\
\hline \multirow[t]{2}{*}{$\mathrm{CS}_{\text {in }}$} & Cont. & $3.00 \pm 0.58$ & $2.71 \pm 0.44$ & $2.41 \pm 0.41$ & $2.13 \pm 0.32$ & $2.08 \pm 0.32$ \\
\hline & \% diff. & $30.6 \pm 8.7 \%$ & $21.5 \pm 4.5 \%$ & $12.4 \pm 4.5 \%$ & $2.0 \pm 3.6 \%$ & $1.5 \pm 4.0 \%$ \\
\hline \multirow[t]{2}{*}{$\mathrm{CS}_{\mathrm{IR}}$} & Cont. & $3.02 \pm 0.48$ & $2.76 \pm 0.42$ & $2.50 \pm 0.43$ & $2.14 \pm 0.32$ & $2.08 \pm 0.32$ \\
\hline & \% diff. & $31.7 \pm 7.3 \%$ & $23.6 \pm 5.3 \%$ & $15.8 \pm 4.7 \%$ & $2.7 \pm 3.9 \%$ & $1.9 \pm 4.1 \%$ \\
\hline \multirow[t]{2}{*}{$\mathrm{TCS}_{n \mathrm{TV}}$} & Cont. & $3.41 \pm 0.45$ & $3.10 \pm 0.38$ & $2.87 \pm 0.36$ & $2.37 \pm 0.30$ & $2.34 \pm 0.29$ \\
\hline & \% diff. & $43.6 \pm 8.8 \%$ & $35.2 \pm 5.9 \%$ & $30.0 \pm 3.8 \%$ & $12.9 \pm 3.4 \%$ & $13.8 \pm 3.7 \%$ \\
\hline \multirow[t]{2}{*}{$\mathrm{TCS}_{n \ell 1}$} & Cont. & $3.03 \pm 0.44$ & $2.73 \pm 0.37$ & $2.48 \pm 0.37$ & $2.21 \pm 0.30$ & $2.17 \pm 0.30$ \\
\hline & $\%$ diff. & $32.4 \pm 6.6 \%$ & $22.8 \pm 4.3 \%$ & $15.2 \pm 2.9 \%$ & $6.0 \pm 3.0 \%$ & $6.1 \pm 3.3 \%$ \\
\hline \multirow[t]{2}{*}{ TCS } & Cont. & $3.46 \pm 0.45$ & $3.15 \pm 0.36$ & $2.97 \pm 0.35$ & $2.47 \pm 0.29$ & $2.45 \pm 0.28$ \\
\hline & \% diff. & $45.0 \pm 8.1 \%$ & $36.9 \pm 6.6 \%$ & $33.4 \pm 4.6 \%$ & $17.0 \pm 3.9 \%$ & $18.5 \pm 4.2 \%$ \\
\hline \multicolumn{7}{|c|}{ Foot Data } \\
\hline \multirow[t]{2}{*}{ ZF } & Cont. & $2.45 \pm 0.23$ & $2.41 \pm 0.20$ & $2.31 \pm 0.19$ & $2.21 \pm 0.16$ & $2.08 \pm 0.15$ \\
\hline & \% diff. & - & - & - & - & - \\
\hline \multirow[t]{2}{*}{$\mathrm{CS}_{\text {low }}$} & Cont. & $2.49 \pm 0.23$ & $2.44 \pm 0.24$ & $2.35 \pm 0.26$ & $2.27 \pm 0.26$ & $2.18 \pm 0.27$ \\
\hline & \% diff. & $1.7 \pm 0.2 \%$ & $1.1 \pm 1.5 \%$ & $1.6 \pm 4.3 \%$ & $2.2 \pm 5.1 \%$ & $4.7 \pm 6.8 \%$ \\
\hline \multirow[t]{2}{*}{$\mathrm{CS}_{\text {high }}$} & Cont. & $3.21 \pm 0.28$ & $2.98 \pm 0.21$ & $2.71 \pm 0.27$ & $2.39 \pm 0.27$ & $2.29 \pm 0.29$ \\
\hline & \% diff. & $27.2 \pm 3.3 \%$ & $21.0 \pm 3.7 \%$ & $15.9 \pm 5.4 \%$ & $7.2 \pm 5.6 \%$ & $9.3 \pm 7.2 \%$ \\
\hline \multirow[t]{2}{*}{$\mathrm{CS}_{\text {int }}$} & Cont. & $3.25 \pm 0.22$ & $3.06 \pm 0.17$ & $2.79 \pm 0.17$ & $2.44 \pm 0.21$ & $2.34 \pm 0.22$ \\
\hline & \% diff. & $28.4 \pm 4.6 \%$ & $23.8 \pm 5.2 \%$ & $19.1 \pm 5.1 \%$ & $9.7 \pm 4.8 \%$ & $12.0 \pm 5.2 \%$ \\
\hline \multirow[t]{2}{*}{$\mathrm{CS}_{\mathrm{IR}}$} & Cont. & $3.41 \pm 0.19$ & $3.14 \pm 0.17$ & $2.83 \pm 0.18$ & $2.45 \pm 0.21$ & $2.34 \pm 0.25$ \\
\hline & \% diff. & $33.1 \pm 5.0 \%$ & $26.3 \pm 6.7 \%$ & $20.6 \pm 5.8 \%$ & $10.1 \pm 5.0 \%$ & $11.6 \pm 6.3 \%$ \\
\hline \multirow[t]{2}{*}{$\mathrm{TCS}_{n \mathrm{TV}}$} & Cont. & $3.49 \pm 0.29$ & $3.30 \pm 0.18$ & $3.18 \pm 0.17$ & $2.65 \pm 0.23$ & $2.58 \pm 0.27$ \\
\hline & \% diff. & $35.2 \pm 4.6 \%$ & $31.1 \pm 6.7 \%$ & $31.9 \pm 6.5 \%$ & $17.9 \pm 6.4 \%$ & $21.3 \pm 7.3 \%$ \\
\hline \multirow[t]{2}{*}{$\mathrm{TCS}_{n \ell 1}$} & Cont. & $3.25 \pm 0.28$ & $3.03 \pm 0.20$ & $2.79 \pm 0.25$ & $2.49 \pm 0.25$ & $2.40 \pm 0.27$ \\
\hline & \% diff. & $28.1 \pm 3.9 \%$ & $22.8 \pm 4.2 \%$ & $18.9 \pm 5.5 \%$ & $11.6 \pm 5.7 \%$ & $14.4 \pm 6.8 \%$ \\
\hline \multirow[t]{2}{*}{ TCS } & Cont. & $3.54 \pm 0.30$ & $3.37 \pm 0.17$ & $3.26 \pm 0.16$ & $2.76 \pm 0.23$ & $2.71 \pm 0.26$ \\
\hline & \% diff. & $36.6 \pm 5.1 \%$ & $33.1 \pm 6.6 \%$ & $34.4 \pm 7.4 \%$ & $22.0 \pm 7.4 \%$ & $26.3 \pm 8.0 \%$ \\
\hline
\end{tabular}

with iterative refinement across both stages. These demanding optimizations can be completed in practical run times using graphical processing units $(39,40)$.

With heavier undersampling, it will become challenging to distinguish vessel signals from aliasing/noise interference. Higher $R$ can be attained for TCS by improving SNR, blood/background contrast and spatial resolution of angiographic acquisitions. These improvements will boost both segmentation and reconstruction performance. Furthermore, increased spatial resolution can also enhance the delineation of vessel boundaries during segmentation. Here we prescribed relatively high spatial resolution (e.g. $0.5 \mathrm{~mm}$ for hand) and used a segmentation that can detect a minimum lumen size equal to this resolution. However, delineation of small, distal vessels might be impaired at more limited spatial resolutions. In such cases, parallel imaging and CS techniques can be combined to alleviate resolution limitations $(41,42)$.

TCS applies first-order finite-difference operators to incur a TV penalty. Penalty weights were kept low here to minimize block artefacts and no significant distortions were observed around vessels. However, higher-order TV terms may enable better denoising in piece-wise smooth regions, while preserving edge information near vessel boundaries (43). Another improvement for TCS concerns the sparsity penalties applied in the image domain. While angiographic images are natively sparse, spatially weighted penalties in relevant sparsifying transform domains (e.g. wavelet domain) might be needed for other applications. Adaptive wavelet-domain penalties have been designed previously based on manual ROI specifications (18) or dependences between wavelet coefficients (44). Similarly, TCS with spatially weighted wavelet penalties may be useful in applications such as coronary imaging.

Residual signals from several background tissues are evident in FIA datasets. First, although synovial fluid in the joints is correctly segmented as part of the background, due to its relatively high $T_{2} / T_{1}$ ratio it yields comparable SSFP signal to vessels. In turn, excessive reconstruction penalties that may introduce significant image artefacts would be required to dampen these bright signals fully. Alternatively, a synovial-fluid map might be generated via a separate segmentation and higher penalty 
weights can then be applied within this map compared with other background tissues. Secondly, the vessel maps presented here contain both arterial and venous streams in the peripheral extremities. Because the two streams may be located closely, segmentation algorithms can assign venous voxels on to arterial vessels (45). For improved separation, segmentation maps can be altered manually to exclude residual venous tissue. Alternatively, the segmentation method can be modified to leverage smoothness of surface curvature and fuzzy connectedness to delineate arterial and venous trees $(26,45,46)$.

In conclusion, the two-step TCS method outperforms regular CS methods in angiographic reconstructions consistently across subjects. While the proposed method was demonstrated successfully for peripheral FIA, it can easily be extended to other angiographic acquisitions. These promising results warrant future studies on a clinical population to examine pathological cases, including abrupt occlusions and small stenotic vessels.

\section{Acknowledgements}

This work was supported in part by a Turkish Scientific and Technical Research Council (TUBITAK) 2232 Fellowship (No: 113C011), a Marie Curie Actions Career Integration Grant (No: PCIG13-GA2013-618101), a European Molecular Biology Organization Installation Grant (No: 3028) and a TUBITAK 3501 Grant (No: 114E546) awarded to TC and in part by a TUBITAK-Slovenia ARRS Collaboration Grant (No: 113E640) awarded to GU. The authors thank Michael Lustig for sharing his reconstruction routines.

\section{REFERENCES}

1 Marckmann P, Skov L, Rossen K, Dupont A, Damholt MB, Heaf JG, Thomsen HS. Nephrogenic systemic _brosis: suspected causative role of gadodiamide used for contrast-enhanced magnetic resonance imaging. J. Am. Soc. Nephrol. 2006; 17: 2359-62.

2 Miyazaki M, Lee VS. Nonenhanced MR angiography. Radiol. 2008; 248: 20-43.

3 Çukur T, Lee JH, Bangerter NK, Hargreaves BA, Nishimura DG. Noncontrast-enhanced owindependent peripheral MR angiography with balanced SSFP. Magn. Reson. Med. 2009; 61: 1533-9.

4 Bangerter NK, Cukur T, Hargreaves BA, Hu BS, Brittain JH, Park D, Gold $\mathrm{GE}$, Nishimura DG. Three-dimensional fluid-suppressed $T_{2^{-}}$prep owindependent peripheral angiography using balanced SSFP. Magn. Reson. Imaging 2011; 29: 1119-24.

5 Çukur T, Shimakawa A, Yu H, Hargreaves BA, Hu BS, Nishimura DG, Brittain JH. Magnetization-prepared IDEAL bSSFP: A owindependent technique for noncontrast-enhanced peripheral angiography. J. Magn. Reson. Imaging 2011; 33: 931-9.

6 Lustig M, Donoho D, Pauly JM. Sparse MRI: The application of compressed sensing for rapid MR imaging. Magn. Reson. Med. 2007; 58: 1182-95.

7 Akçakaya M, Hu P, Chuang ML, Hauser TH, Ngo LH, Manning WJ, Tarokh V, Nezafat R. Accelerated noncontrast-enhanced pulmonary vein MRA with distributed compressed sensing. J. Magn. Reson. Imaging 2011; 33: 1248-55.

8 Çukur T, Lustig M, Nishimura DG. Improving noncontrast- enhanced steady-state free precession angiography with compressed sensing. Magn. Reson. Med. 2009; 61: 1122-31.

9 Trzasko JD, Haider CR, Borisch EA, Campeau NG, Glockner JF, Riederer SJ, Manduca A. Sparse-CAPR: highly accelerated 4D CE-MRA with parallel imaging and nonconvex compressive sensing. Magn. Reson. Med. 2011; 66: 1019-32.

$10 \mathrm{Wu} \mathrm{H}$, Block WF, Samsonov AA. HYPRconstrained compressed sensing reconstruction for accelerated time resolved imaging. In: Proc. 16th Annual Meeting of ISMRM, International Society for Magnetic Resonance in Medicine (ISMRM): Toronto; 2008; 339.

11 Block KT, Uecker M, Frahm J. Undersampled radial MRI with multiple coils: Iterative image reconstruction using a total variation constraint. Magn. Reson. Med. 2007; 57: 1086-98.
12 Gamper U, Boesiger P, Kozerke S. Compressed sensing in dynamic MRI. Magn. Reson. Med. 2008; 59: 365-73.

13 Haldar JP, Hernando D, Song S, Liang Z. Anatomically constrained reconstruction from noisy data. Magn. Reson. Med. 2008; 59: 810-8.

14 Gong E, Huang F, Ying K, Wu W, Wang S, Yuan C. PROMISE: parallelimaging and compressed-sensing reconstruction of multicontrast imaging using SharablE information. Magn. Reson. Med. 2015; 73: 523-35.

15 Liang D, DiBella EVR, Chen RR, Ying L. k-t ISD: dynamic cardiac MR imaging using compressed sensing with iterative support detection. Magn. Reson. Med. 2012; 68: 41-53.

16 Johnson KM, Velikina J, Wu Y, Kecskemeti S, Wieben O, Mistretta CA. Improved waveform fidelity using local HYPR reconstruction (HYPR LR). Magn. Reson. Med. 2008; 59: 456-62.

17 Stinson EG, Borisch EA, Johnson CP, Trzasko JD, Young PM, Riederer SJ. Vascular masking for improved unfolding in 2D SENSE-accelerated 3D contrast-enhanced MR angiography. J. Magn. Reson. Imaging 2014; 39: 1161-70.

$18 \mathrm{Oh} \mathrm{H}$, Lee $\mathrm{S}$. Visually weighted reconstruction of compressive sensing MRI. Magn. Reson. Imaging 2014; 32: 270-80.

19 Zhang C, van de Giessen M, Eisemann E, Vilanova A. User-guided compressed sensing for magnetic resonance angiography. In: Engineering in Medicine and Biology Society (EMBC), 2014 36th Annual International Conference of the IEEE, Institute of Electrical and Electronics Engineers (IEEE): Chicago; 2014; 2416-2419.

20 Konar AS, Aiholli S, Shashikala HC, Ramesh-Babu DR, Geethanath S. Application of region of interest compressed sensing to accelerate magnetic resonance angiography. Conf. Proc. IEEE Eng. Med. Biol. Soc. 2014; 2428-31.

21 Hutter J, Grimm R, Forman C, Hornegger J, Schmitt P. Vessel adapted regularization for iterative reconstruction in MR angiography. In: Proc. 20th Annual Meeting of Int. Soc. Magn. Reson. Med., International Society for Magnetic Resonance in Medicine (ISMRM): Melbourne; 2012; 2541.

22 Cetin S, Unal G. A higher-order tensor vessel tractography for segmentation of vascular structures. IEEE Trans. Med. Imaging 2015; 34: 2172-85.

23 Hargreaves BA, Vasanawala SS, Pauly JM, Nishimura DG. Characterization and reduction of the transient response in steady-state MR imaging. Magn. Reson. Med. 2001; 46: 149-58.

24 Çukur T, Lustig M, Saritas EU, Nishimura DG. Signal compensation and compressed sensing for magnetization-prepared MR angiography. IEEE Trans. Med. Imaging 2011; 34: 107-15.

25 Stefancik RM, Sonka M. Highly automated segmentation of arterial and venous trees from three-dimensional magnetic resonance angiography (MRA). Int. J. Cardiovasc. Imaging 2001; 17: 37-47.

26 Tizon X, Smedby O. Segmentation with gray-scale connectedness can separate arteries and veins in MRA. J. Magn. Reson. Imaging 2002; 15: 438-45.

27 Chapman BE, Stapelton JO, Parker DL. Intracranial vessel segmentation from time-of-ight MRA using pre-processing of the MIP Z-buffer: accuracy of the ZBS algorithm. Med. Image Anal. 2004; 8: 113-26.

28 de Koning PJH, Schaap JA, Janssen JP, Westenberg JJM, van der Geest RJ, Reiber JHC. Automated segmentation and analysis of vascular structures in magnetic resonance angiographic images. Magn. Reson. Med. 2003; 50: 1189-98.

29 Müller-Eschner M, Müller T, Biesdorf A, Wörz S, Rengier F, Böckler D, Kauczor HU, Rohr K, von Tengg-Kobligk H. 3D morphometry using automated aortic segmentation in native MR angiography: an alternative to contrast enhanced MRA? Cardiovasc. Diagn. Ther. 2014; 4: 80-7.

30 Cetin S, Demir A, Yezzi A, Degertekin M, Unal G. Vessel tractography using an intensity based tensor model with branch detection. IEEE Trans. Med. Imaging 2013; 32: 348-63.

31 Cetin S, Unal G. Higher order tensor-based segmentation and $n$ furcation modeling of vascular structures. In: Proc. Joint MICCAI Workshop CVII STENT, Computing and Computer Assisted Intervention (MICCAl) Society: Boston; 2014; 68.

32 Yip E, Yun J, Wachowicz K, Heikal AA, Gabos Z, Rathee S, Fallone BG. Prior data assisted compressed sensing: A novel MR imaging strategy for real time tracking of lung tumors. Med. Phys. 2014; 41: 082301.

33 Ye JC, Tak S, Han Y, Park HW. Projection reconstruction MR imaging using FOCUSS. Magn. Reson. Med. 2007; 57: 764-75.

34 Greenman RL, Shirosky JE, Mulkern RV, Rofsky NM. Double inversion black-blood fast spinecho imaging of the human heart: A comparison between 1.5 T and 3.0 T. J. Magn. Reson. Imaging 2003; 17: 648-55. 
35 Bernstein MA, King KF, Zhou XJ. Handbook of MRI Pulse Sequences, 1st edn. Elsevier Academic Press: Burlington, MA; 2004.

36 Mazhbich Bl. Noninvasive determination of elastic properties and diameter of human limb arteries. Pugers Arch. 1983; 396: 254-9.

37 Constantinides CD, Atalar E, McVeigh ER. Signalto- noise measurements in magnitude images from Copyright c 2016 John Wiley \& Sons, Ltd. E. ILICAK ET AL. NMR phased arrays. Magn. Reson. Med. 1997; 38: 852-7.

38 Zhang Q, Plemmons R, Kittle D, Brady D, Prasad S. Joint segmentation and reconstruction of hyperspectral data with compressed measurements. Appl. Optics 2011; 50: 4417-35.

39 Murphy M, Alley M, Demmel J, Keutzer K, Vasanawala S, Lustig M. Fast $\ell_{1}$-SPIRiT compressed sensing parallel imaging MRI: scalable parallel implementation and clinically feasible runtime. IEEE Trans. Med. Imaging 2012; 31: 1250-62.

40 Nam S, Akçakaya M, Basha T, Stehning C, Manning WJ, Tarokh V, Nezafat R. Compressed sensing reconstruction for whole-heart imaging with 3D radial trajectories: a graphics processing unit implementation. Magn. Reson. Med. 2013; 69: 91-102.

41 Otazo R, Kim D, Axel L, Sodickson DK. Combination of compressed sensing and parallel imaging for highly accelerated first-pass cardiac perfusion MRI. Magn. Reson. Med. 2010; 64: 767-76.
42 Liang D, Liu B, Wang J, Ying L. Accelerating SENSE using compressed sensing. Magn. Reson. Med. 2009; 62: 1574-84.

43 Knoll F, Bredies K, Pock T, Stollberger R. Second order total generalized variation (TGV) for MRI. Magn. Reson. Med. 2011; 65: 480-91.

44 Akçakaya M, Nam S, Hu P, Moghari MH, Ngo LH, Tarokh V, Manning WJ, Nezafat R. Compressed sensing with wavelet domain dependencies for coronary MRI: a retrospective study. IEEE Trans. Med. Imaging 2011; 30: 1090-9.

45 van Bemmel CM, Spreeuwers LJ, Viergever MA, Niessen WJ. Level-setbased artery-vein separation in blood pool agent CE-MR angiograms. IEEE Trans. Med. Imaging 2003; 22: 1224-34.

46 Lei T, Udupa JK, Saha PK, Odhner D. Arteryvein separation via MRA an image processing approach. IEEE Trans. Med. Imaging 2001; 20: 689-703.

\section{SUPPORTING INFORMATION}

Additional supporting information can be found in the online version of this article at the publisher's website. 